\title{
Autoimmunerkrankungen der Leber
}

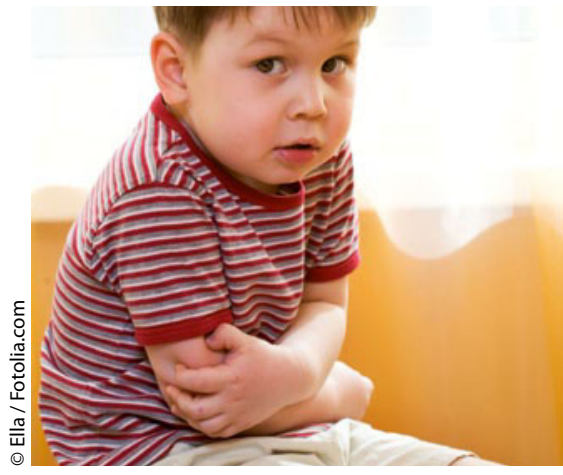

$\mathrm{Zu}$ den wichtigsten Erkrankungen der Leber bzw. der Gallenwege mit vermuteter autoimmuner Genese zählen die Autoimmunhepatitis (AIH) und die sklerosierende Cholangitis (SC), das „Overlap-Syndrom“ ist eine Mischform der beiden Erkrankungen.

Prof. Dr. Michael Melter aus Regensburg wies auf eine Reihe von Autoantikörpern hin, die bei der AIH nachgewiesen werden können: Antikörper gegen glatte Muskulatur (SMA), antinukleäre Antikörper (ANA), mikrosomale Anti- gene von Leber und Niere (LKM-1) oder gegen lösliches Leberantigen (SLA). Die Symptomatik reicht von der akuten Hepatitis über eine progressive Erschöpfung („Typ Mimikry“) bis zur portalen Hypertension. Bis zu 15\% können asymptomatisch verlaufen.

Bei der SC sind perinukleäre antineutrophile Antikörper (pANCA), aber auch ANA oder SMA nachweisbar. In bis zu 90\% besteht eine Hepato(spleno)megalie, in der Hälfte der Fälle Ikterus.

Therapie der Wahl bei der AIH ist die Immunsuppression, initial mit Prednisolon oder Budesonid. Auch Azathioprin, Mycophenolat-Mofetil oder Cyclosporin A werden eingesetzt. Die Evidenzlage bei der SC dagegen ist schlecht. Hier kommt symptomatisch Ursodeoxycholsäure zum Einsatz. Bei beiden Erkrankungen kann im fortgeschrittenen Stadium mit Fibrose/ Zirrhose eine Lebertransplantation notwendig werden.

Dr. Andreas Fischer

Melter M. Autoimmunerkrankungen der Leber und Gallenwege bei Kindern und Jugendlichen

\section{Buchtipp}

\section{Meisterwerke aus der Psychiatrie}

Der Psychiater und Kunsthistoriker Hans Prinzhorn (1886-1933) hatte neben seiner Tätigkeit an der Universität Heidelberg die Lehrsammlung der Patientenkunst zu betreuen. Mit großem Einsatz sammelte er deutschlandweit Werke psychisch Kranker.

Sein Buch „Bildnerei der Geisteskranken“ ist in drei Abschnitte gegliedert. Im ersten wird eine eigene Ausdruckstheorie der bildenden Kunst entwickelt. Prinzhorn war überzeugt, dass die Patienten in ihren Bildern Unbewusstes darstellen. „Sie wissen nicht, was sie tun." Der zweite Abschnitt stellt zehn schizophrene Meister mit ihren Biografien und der ausführlichen Betrachtung ihrer Werke vor. Der dritte Abschnitt behandelt grundlegende, vor allem psychiatrische und kunstkritische Fragen. Es ging Prinzhorn nicht um einen diagnostischen Nutzen der Werke (diesen bestreitet er sogar), sondern um die ästhetische Wertschätzung der Bilder. Die „Bildnerei“ beeinflusste Künstler der expressionistischen wie auch der surrealistischen Malerei.

Ab 1933 gerieten in Deutschland Werke von Anstaltsinsassen ins Visier der Nationalsozialisten. Diese Bilder dienten als Vergleichsmaterial, um die Werke anderer „entarteter" Künstler zu diffamieren. Erst in den 1950er-Jahren wurde die Kunst der psychiatrisch Erkrankten wieder entdeckt. Das Buch von Hans Prinzhorn ist jetzt zum siebten Mal seit der Erstauflage im Jahr 1922 erschienen. Dr. Kirsten Stollhoff

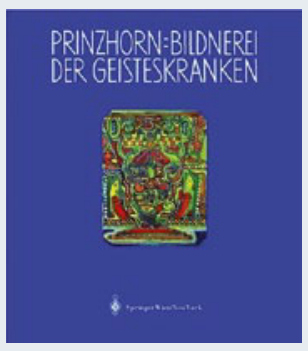

Hans Prinzhorn

Bildnerei der Geisteskranken Springer-Verlag, Heidelberg, 2011 $48,59 €$ ISBN 978-3-70910720-1 